\title{
Application of Multi-Scale Fusion Attention U-Net to Segment the Thyroid Gland on CT Localization Images for Radiotherapy
}

\section{Xiaobo Wen}

the Third Affiliated Hospital of Kunming Medical University

\section{Biao Zhao}

the Third Affiliated Hospital of Kunming Medical University

\section{Meifang Yuan}

the Third Affiliated Hospital of Kunming Medical University

Jinzhi Li

the Third Affiliated Hospital of Kunming Medical University

\section{Mengzhen Sun}

the Third Affiliated Hospital of Kunming Medical University

\section{Lishuang Ma}

the Third Affiliated Hospital of Kunming Medical University

\section{Chaoxi Sun}

the Third Affiliated Hospital of Kunming Medical University

Yi Yang ( $\nabla$ yiyangrt@126.com )

the Third Affiliated Hospital of Kunming Medical University

\section{Research Article}

Keywords: U-net model, Multi-scale Fusions, medical image segmentation, thyroid, radiotherapy, cSE

Posted Date: October 5th, 2021

DOI: https://doi.org/10.21203/rs.3.rs-949323/v1

License: (c) (1) This work is licensed under a Creative Commons Attribution 4.0 International License. Read Full License 


\section{Application of Multi-scale Fusion Attention U-net to segment the thyroid gland on CT localization images for radiotherapy}

Xiaobo Wen ${ }^{1}$, Biao Zhao ${ }^{1}$, Meifang Yuan ${ }^{1}$, Jinzhi Li ${ }^{1}$, Mengzhen Sun ${ }^{1}$, Lishuang MA ${ }^{1}$, Chaoxi Sun ${ }^{2}$, Yi Yang ${ }^{1}$

1. Department of Radiotherapy, Yunnan Cancer Hospital, the Third Affiliated Hospital of Kunming Medical University, Kunming, Yunnan, China.

2. Department of Neurosurgery, Yunnan Cancer Hospital, the Third Affiliated Hospital of Kunming Medical University, Kunming, Yunnan, China.

\section{Corresponding Authors:}

Yi Yang, Department of Radiotherapy, Yunnan Cancer Hospital, the Third Affiliated Hospital of Kunming Medical University, 519 Kunzhou Road, Xishan District, Kunming City 650118, Yunnan Province, China.

E-mail: yiyangrt@126.com 
3

\section{Abstract}

Objectives To explore the performance of Multi-scale Fusion Attention U-net (MSFA-U-net) in thyroid gland segmentation on CT localization images for radiotherapy.

Methods CT localization images for radiotherapy of 80 patients with breast cancer or head and neck tumors were selected; label images were manually delineated by experienced radiologists. The data set was randomly divided into the training set $(n=60)$, the validation set $(n=10)$, and the test set $(n=10)$. Data expansion was performed in the training set, and the performance of the MSFA-U-net model was evaluated using the evaluation indicators Dice similarity coefficient (DSC), Jaccard similarity coefficient (JSC), positive predictive value (PPV), sensitivity (SE), and Hausdorff distance (HD).

Results With the MSFA-U-net model, the DSC, JSC, PPV, SE, and HD indexes of the segmented thyroid gland in the test set were $0.8967 \pm 0.0935,0.8219 \pm 0.1115,0.9065 \pm 0.0940,0.8979 \pm 0.1104$, and 2.3922 \pm 0.5423 , respectively. Compared with U-net, HR-net, and Attention U-net, MSFA-U-net showed that DSC increased by $0.052,0.0376$, and 0.0346 respectively; JSC increased by 0.0569 , 0.0805 , and 0.0433 , respectively; SE increased by $0.0361,0.1091$, and 0.0831 , respectively; and HD increased by $-0.208,-0.1952$, and -0.0548 , respectively. The test set image results showed that the thyroid edges segmented by the MSFA-U-net model were closer to the standard thyroid delineated by the experts, in comparison with those segmented by the other three models. Moreover, the edges were smoother, over-anti-noise interference was stronger, and oversegmentation and undersegmentation were reduced. Conclusion The MSFA-U-net model can meet basic clinical requirements and improve the efficiency of physicians' clinical work. 
Keywords: U-net model; Multi-scale Fusions; medical image segmentation; thyroid; radiotherapy; cSE

25

\section{Introduction}

Head and neck tumors and breast cancer are currently the tumors with the highest morbidity and mortality worldwide(1). In 2020, there were 19.29 million new cancer cases worldwide, of which 4.57 million cases $(23.7 \%)$ were in China. Radiotherapy is an effective and common method for the treatment of head and neck cancer and breast cancer (2-4). Accurately delineating organs at risk (OARs) when designing radiotherapy plans can effectively avoid radiation side effects. At present, the outline of OARs is mainly done by physicians; thus, the process is subjective, time-consuming, and labor-intensive.

With the rapid development of artificial intelligence, Ronneberger (5) and others proposed the Unet neural network model in 2015. The segmentation and delineation method based on deep learning has gradually been developed and applied in clinical work(6-10). Ye et al.(7) used an improved model, a dense connectivity embedding U-net, to train and segment the T1 and T2 MRI images of 44 patients with nasopharyngeal carcinoma; the authors obtained DSC of 0.87 after 10-time cross-validation. However, in segmentation studies on OARs for head and neck tumors and breast cancer, the thyroid gland has often been ignored and has not been considered an OAR for automatic segmentation studies (8); therefore, the automatic delineation of the thyroid gland in CT localization images for radiotherapy has rarely been studied. It has been shown that side effects, such as thyroid function decline, occur when the radiation dose of the thyroid gland exceeds 26 Gy (11). Franco and others (12) conducted a retrospective study on 3-dimensional conformal radiation therapy (3D-CRT) for breast cancer. They 
found that in about $45 \%$ of patients with lymph node-positive breast cancer, the thyroid gland was exposed to a radiation dose higher than $26 \mathrm{~Gy}$. Other studies have shown $(13,14)$ that, at $5-10$ years after receiving radiotherapy, the incidence of hypothyroidism in patients with nasopharyngeal cancer or breast cancer is $20 \%$ to $52 \%$; moreover, the incidence of hypothyroidism increases with the increasing time of follow-up. Therefore, during radiotherapy planning, it is necessary to limit the radiation of the thyroid gland. Considering that CT localization for radiotherapy involves a simulated-positioning largeaperture CT (Somatom Sensation Open, 24 rows, $\Phi 85 \mathrm{~cm}$ ), which is limited by small size and poor image resolution, automatic segmentation of the thyroid gland based on deep learning model is difficult. It is necessary to further explore the performance of the deep learning model on CT localization images for radiotherapy. This study proposed a model that combines cSE attention mechanism and HR-net on the basis of U-net, and applied it to segment the thyroid gland on CT localization images so as to assist with the delineation of the thyroid gland as an OAR in radiotherapy.

\section{Materials and methods}

\subsection{Data set acquisition}

The experimental data set of this study was obtained from 80 patients with nasopharyngeal carcinoma or breast cancer admitted to the same attending group at the Department of Radiotherapy of Yunnan Cancer Hospital from June 2014 to April 2019. Siemens large-aperture CT (Sensation Open 24 CT) was used to perform the simulated localization for each patient. The slices were $5 \mathrm{~mm}$ or $3 \mathrm{~mm}$ thick, and the images of $512 \times 512$ were obtained in DICOM format. The mask images were drawn by senior radiotherapy physicians using the 3D slicer software. After drawing, the mask images in DICOM format were converted to PNG format; the result is shown in Figure 1. 


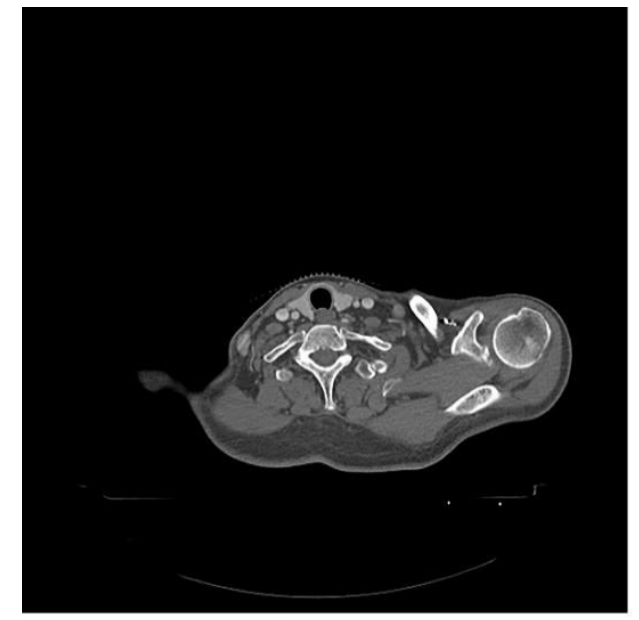

a. CT image

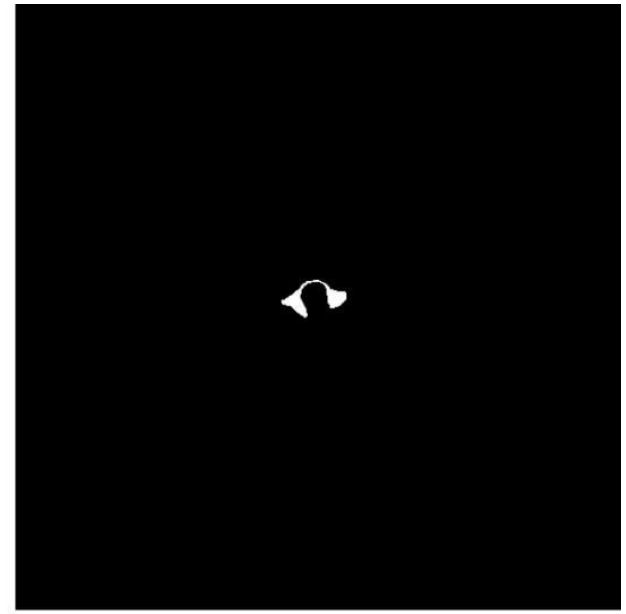

b. ground truth

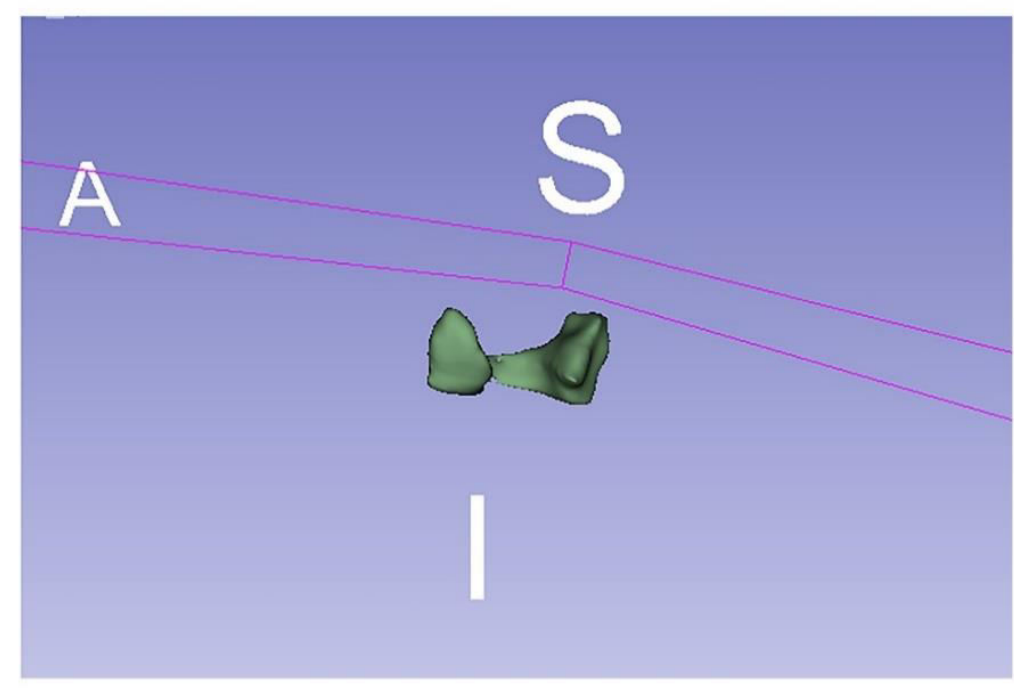

\section{c. 3D-image}

67

Fig 1. CT localization image, label image, and 3D image (a. a standard image of the imported model (CT image), b. the corresponding label image (mask), c. the thyroid gland drawn in 3D)

\section{The data set was divided $(6: 1: 1)$ into the training set, the validation set, and the test set. Due to the} small number of medical data sets and the high cost of drawing, it was difficult to collect a large number of data sets; however, if the training data are not large enough, there is a risk of overfitting the model. Therefore, in this study, data expansion was conducted by means of rotation, flipping, zooming, 
and shearing, so as to increase the training sample size in the training set and avoid overfitting.

\subsection{Data set preprocessing}

To better highlight the region of interest (ROI), we first adopted HU value conversion to convert image pixels into HU values and then adjusted the window width and window level of the converted data to highlight the thyroid gland; finally, we used adaptive histogram equalization to further enhance the contrast (contrast) and normalize the images (normalization).

\subsection{Model framework}

The model in this study was improved on the basis of the U-net and HR-net model architectures.

The main improvement in this study included the replacement of the two feature extraction convolutions of different resolutions in the U-net downsampling process with multiple convolution blocks in HR-net and feature fusion between different scales, and introduction of Spatial Squeeze and Channel Excitation Block (cSE) attention mechanism (15), called MSFA-U, into each convolution block, as shown in Figure 2. In the downsampling process of the model, we connected an cSE module after extracting two $3 \times 3$ convolutional features and fused the input features with the features after the scale operation by means of residual connection. The residual connection consisted of a $1 \times 12 \mathrm{~d}$ convolution and a normalization layer(16) (Batch Normalization, BN). In the cSE module, we used a global average pooling layer (GlobalAveragePooling, GAP) to convert a feature map from Channel $\times$ height $\times$ width to Channel $\times 1 \times 1$ and then used Dense to reduce the feature channel by half, which was achieved by activating the function Relu. After that, the feature channel was restored to normal size by using Dense, and the function Sigmoid was used to activate the channel. Finally, the calibrated 
feature map was obtained through the channel-wise multiplication. The schematic diagram of the residual connection and cSE module structure is shown in Figure 3. The residual connection effectively prevents the model from disappearing and exploding with the deepening of the network(17). Moreover, the cSE module is able to effectively reflect the relationship between different channels and assign different weights so that the model can focus on important features that accurately segment the thyroid gland during the training process. The whole module is called an Attention Resblock (Figures 2 and 3).

The traditional U-net model uses the maximum pooling layer to perform downsampling and reduce the amount of parameters. This method may lead to the loss of information during the feature extraction process. Therefore, in this study, the stepped convolution was used to perform the downsampling. Stride convolution can remove redundant information, thereby reducing the size of the feature map. The model uses multiple branches of different resolutions to extract features in parallel during the training process, and it performs feature fusion between different scales after each attention residual block so as to achieve strong semantic information and precise location during the training process. One or more transposed convolutions $(3 \times 3)$ are used in the conversion from low resolution to high resolution, while one or more stride convolutions $(3 \times 3)$ are used in the conversion from high resolution to low resolution, as shown in Fig. 3 In the upsampling part, the attention residual block replaces the two convolution operations in U-net to avoid excessive parameters. Meanwhile, dropout layer is added after each shortcut connection (the parameter set to 0.2 ) to avoid the decrease of generalization caused by the overfitting resulting from multiple feature fusions between different scales during the training process. 


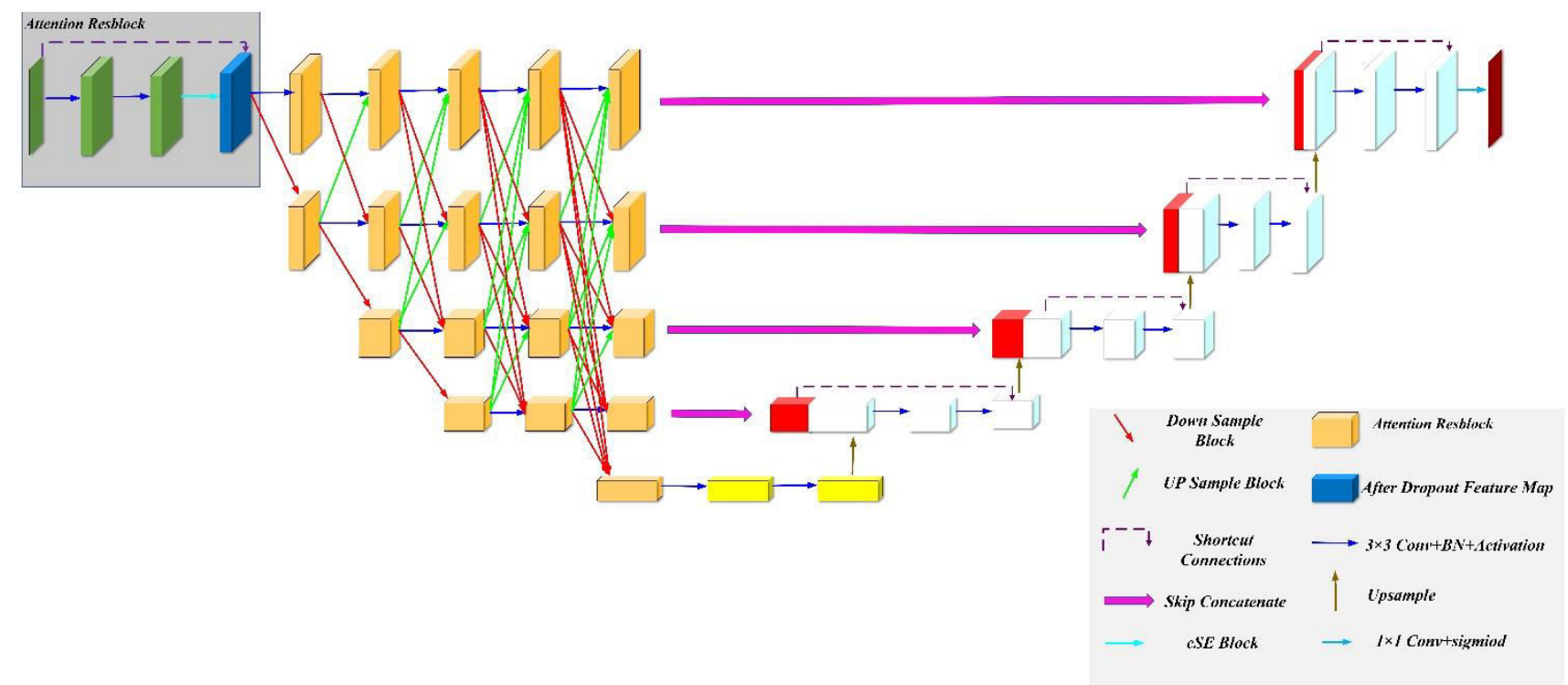

Fig. 2 MSFA-U-net structure

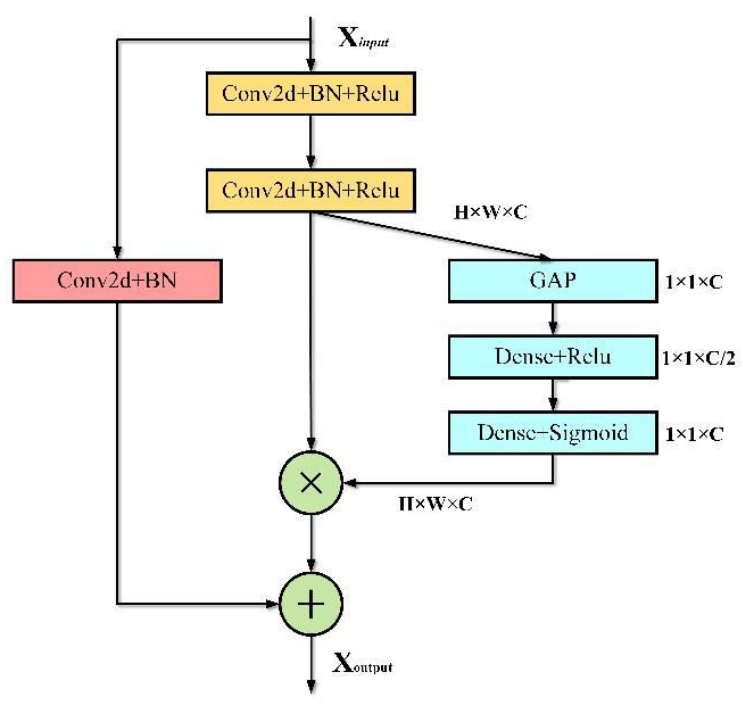

a. Attention Resblock Module

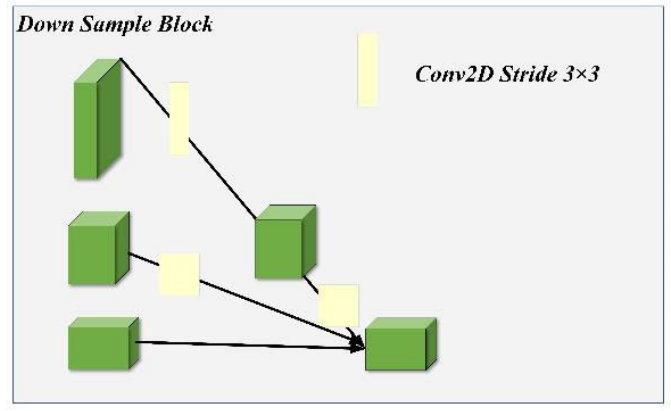

b. High resolution to a low resolution

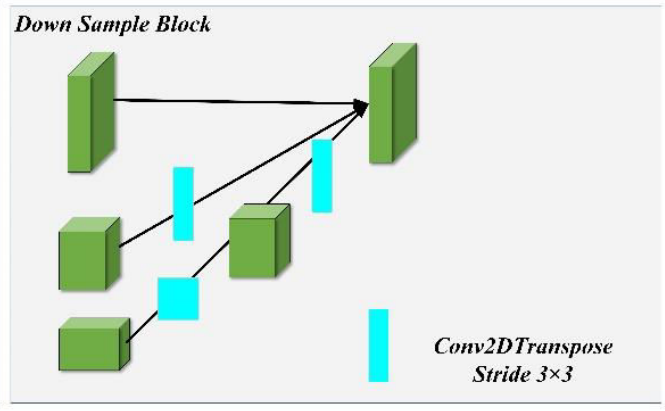

c. Low resolution to a High resolution

Fig. 3 Attention Resblock Module and Feature fusion of different scales 
Resblock module; b. one or more stride convolutions $(3 \times 3)$ are used in the conversion from

high resolution to low resolution; c. one or more transposed convolutions $(3 \times 3)$ are used in

\subsection{Model operating environment and parameters}

Table 1 Network training parameters

\begin{tabular}{|c|c|c|c|c|c|c|}
\hline \multirow[b]{2}{*}{ Model } & \multirow[b]{2}{*}{ Batch Size } & \multicolumn{2}{|r|}{ Image Size } & & \multirow[b]{2}{*}{ Decay Steps } & \multirow[b]{2}{*}{ Decay_Rate } \\
\hline & & Epoch & (Pixels) & Learning Rate & & \\
\hline HR-net & 2 & 120 & $512 \times 512$ & $8 e-5$ & 300 & 0.96 \\
\hline MSFA-U-net & 2 & 120 & $512 \times 512$ & $2 \mathrm{e}-4$ & 300 & 0.96 \\
\hline
\end{tabular}



the traditional cross-entropy loss function would make the model more inclined to predict the the model prediction and the real segmentation, thereby effectively solving the problem of serious thyroid imbalance. The formula is shown in 1.1:

$$
D L=1-2 \frac{|X \cap Y|+\varepsilon}{|X|+|Y|+\varepsilon},
$$

where $\mathrm{X}$ represents the label matrix of the real thyroid gland, $\mathrm{Y}$ is the prediction matrix of the model predicting the thyroid gland, and $\varepsilon$ represents a constant included to avoid being divided by zero.

\subsection{Evaluation indexes} values closer to 1 indicate better prediction. 
The calculation of $\operatorname{PPV}(21)$ and $\mathrm{SE}(22)$ is shown in formulas (1.4) and (1.5):

$$
\begin{aligned}
& P P V=\frac{T P}{T P+F P}, \\
& S E=\frac{T P}{T P+F N},
\end{aligned}
$$

where TP represents the foreground target value that is predicted correctly, and FP represents the foreground target value that is predicted incorrectly, and FN represents the background target value that is predicted incorrectly.

$\mathrm{HD}(23)$ calculation is shown in formula (1.6):

$$
H(X, Y)=\max (h(X, Y), h(Y, X)),
$$

where $h(X, Y)=\operatorname{maxmin}_{x \in X}\|a-b\| h(Y, X)=\operatorname{maxmin}_{y \in Y}\|a-b\|$. Smaller values of HD indicate better prediction.

\section{Experimental results}

\subsection{Comparison model design}

In order to prove the validity of the proposed MSFA-U-net model, we selected three model architectures related to MSFA-U-net and conducted comparative experiments:

1) U-net(5): U-shaped symmetrical structure, composed of upsampling, downsampling, and skip connection. The skip connection effectively combines the feature information between different resolutions and makes up for the loss of the high-resolution features in the downsampling process, and it can output the feature map more accurately. The U-net model is one of the models widely used in the medical field.

2) HR-net(24): This model maintains high-resolution output during the feature extraction process. It has multiple parallel subnets with different resolutions to compress and extract features, and perform 
multiple different scale feature fusion to get richer high-resolution features. In the original study, the author used bilinear interpolation upsampling. In order to better extract features for fusion, in this study, we used transposed convolution to convert from low resolution to high resolution.

3) Attention U-net(25): This model introduces an attention-gating mechanism, so that the information in the jump connection of the U-net model has different weights and pays more attention to the ROI area.

\subsection{Qualitative analysis of results}

Figure 4 shows the results of the segmentation label map of the four models in the test set of the thyroid gland, and Figure 5 shows the coverage map of the four models on the CT image of the radiotherapy location. Given that there are a large number of blood vessels and soft tissues with similar gray levels around the thyroid gland(26), oversegmentation and undersegmentation are expected in the edge segmentation. As shown in the segmented label map in Figure 4, a part of the surrounding blood vessels and soft tissues was mistakenly segmented as the part of the left lobe of the thyroid gland when the U-net model segmented the left lobe of the thyroid gland. As for HR-net and Attention U-net, although oversegmentation of the surrounding soft tissues and blood vessels decreased, there were still some noise points and uneven edges. However, the MSFA-U-net architecture used in the study achieved smooth edges and decreased noise. Moreover, although we adjusted the window width and window level and adopted adaptive contrast enhancement, some lesions at some levels of the thyroid may have resulted in less obvious enhancement. In the thyroid segmentation at these levels, MSFA-Unet exhibited better robustness than the other three models. In summary, compared with the other three models, MSFA-U-net improved the performance of the thyroid gland segmentation on CT localization 

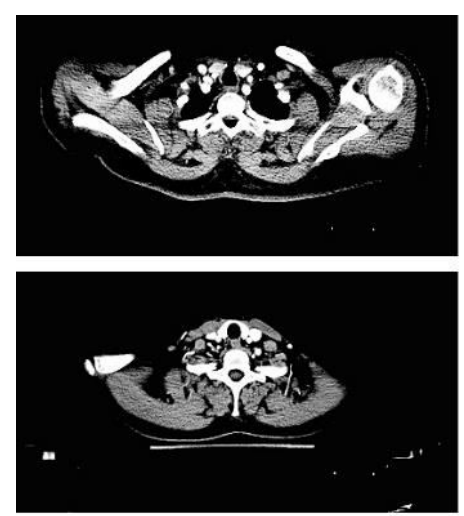

a.CT image
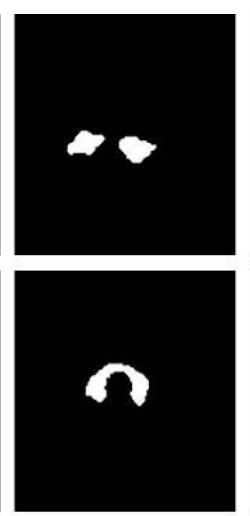

b.ground truth
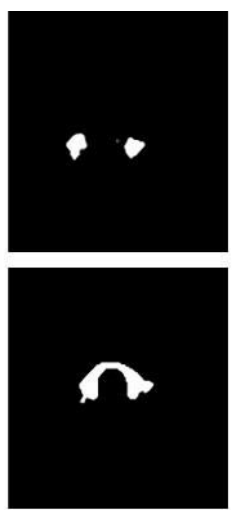

c.U-net segmentation
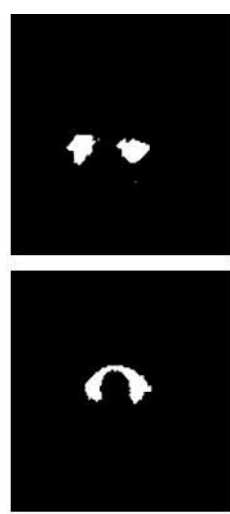

I.HR-net segmentation
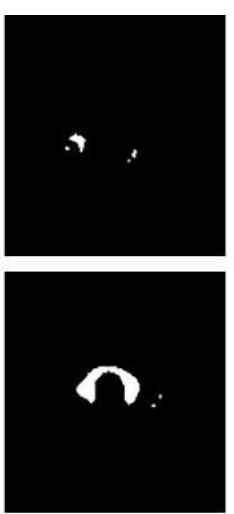

Attention $U$-net
segmentation
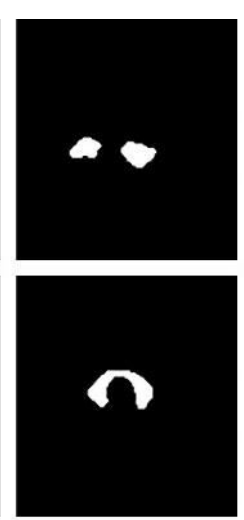

f.MSFA-U-net segmentation

Fig. 4 Thyroid gland segmentation of the four models on CT localization images for

radiotherapy
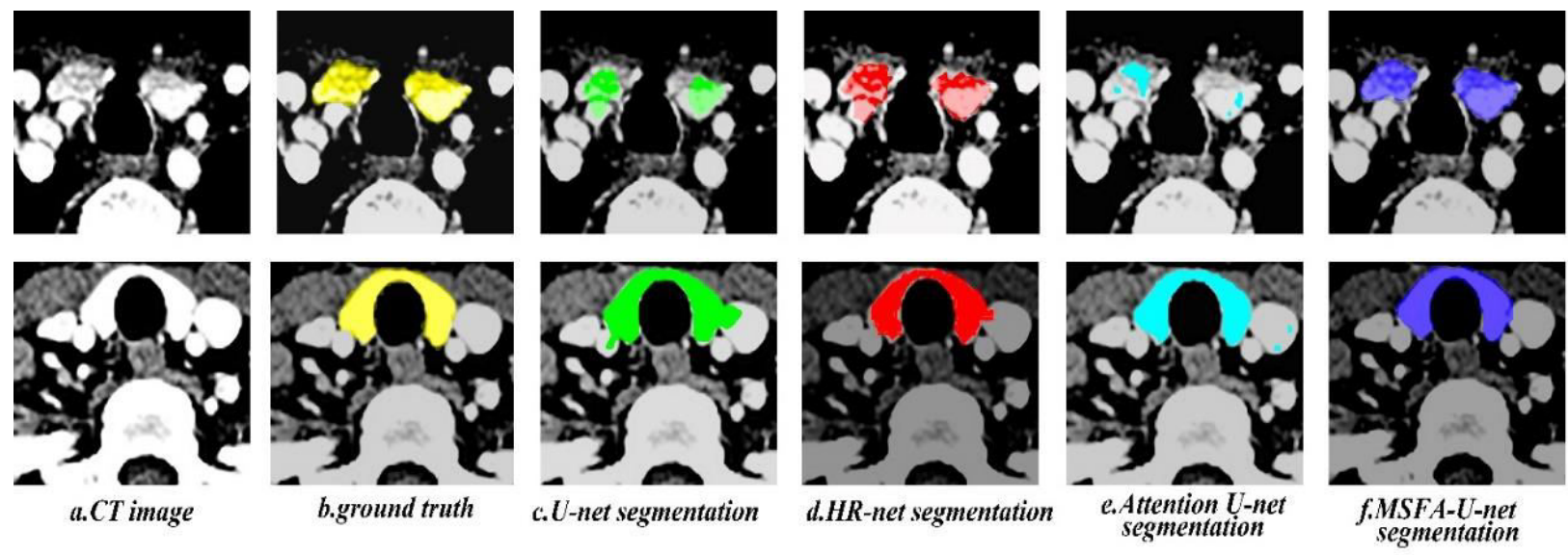

Fig. 5 The thyroid coverage map of the four models on CT localization images for

radiotherapy

\subsection{Quantitative analysis of the results}


221 image segmentation models, MSFA-U-net achieved great improvement in multiple evaluation 222 indicators: DSC improvement range, [0.0346, 0.052]; JSC improvement range [0.0433, 0.0805]; SE 223 improvement range [0.0361, 0.1091]; and HD improvement range $[-0.208,-0.0548]$. As for the PPV evaluation index, MSFA-U-net was better than the U-net model and worse than the HR-net and Attention U-net models; however, as for the other evaluation indexes, HR-net and Attention U-net models were worse than the MSFA-U -net.

Table 2 Assessment indexes of the test set $(\bar{x} \pm s)$

\begin{tabular}{ccccc} 
& U-net & HR-net & Attention U-net & MSFA-U-net \\
\hline DSC & $0.8591 \pm 0.1046$ & $0.8447 \pm 0.0938$ & $0.8621 \pm 0.1502$ & $\mathbf{0 . 8 9 6 7} \pm \mathbf{0 . 0 9 3 5}$ \\
\hline JSC & $0.7650 \pm 0.1337$ & $0.7414 \pm 0.1273$ & $0.7786 \pm 0.1633$ & $\mathbf{0 . 8 2 1 9} \pm \mathbf{0 . 1 1 1 5}$ \\
\hline PPV & $0.8775 \pm 0.1179$ & $0.9257 \pm 0.0759$ & $\mathbf{0 . 9 5 2 3} \pm \mathbf{0 . 0 6 8 9}$ & $0.9065 \pm 0.0940$ \\
\hline SE & $0.8618 \pm 0.1212$ & $0.7888 \pm 0.1276$ & $0.8148 \pm 0.1683$ & $\mathbf{0 . 8 9 7 9} \pm \mathbf{0 . 1 1 0 4}$ \\
\hline HD & $2.6002 \pm 0.5731$ & $2.5874 \pm 0.5412$ & $2.4470 \pm 0.6872$ & $\mathbf{2 . 3 9 2 2} \pm \mathbf{0 . 5 4 2 3}$ \\
\hline
\end{tabular}

* Bold numbers mean optimal value.

\subsection{Box plot of the results}

To further evaluate the differences between the four models, we made box plots of the evaluation 

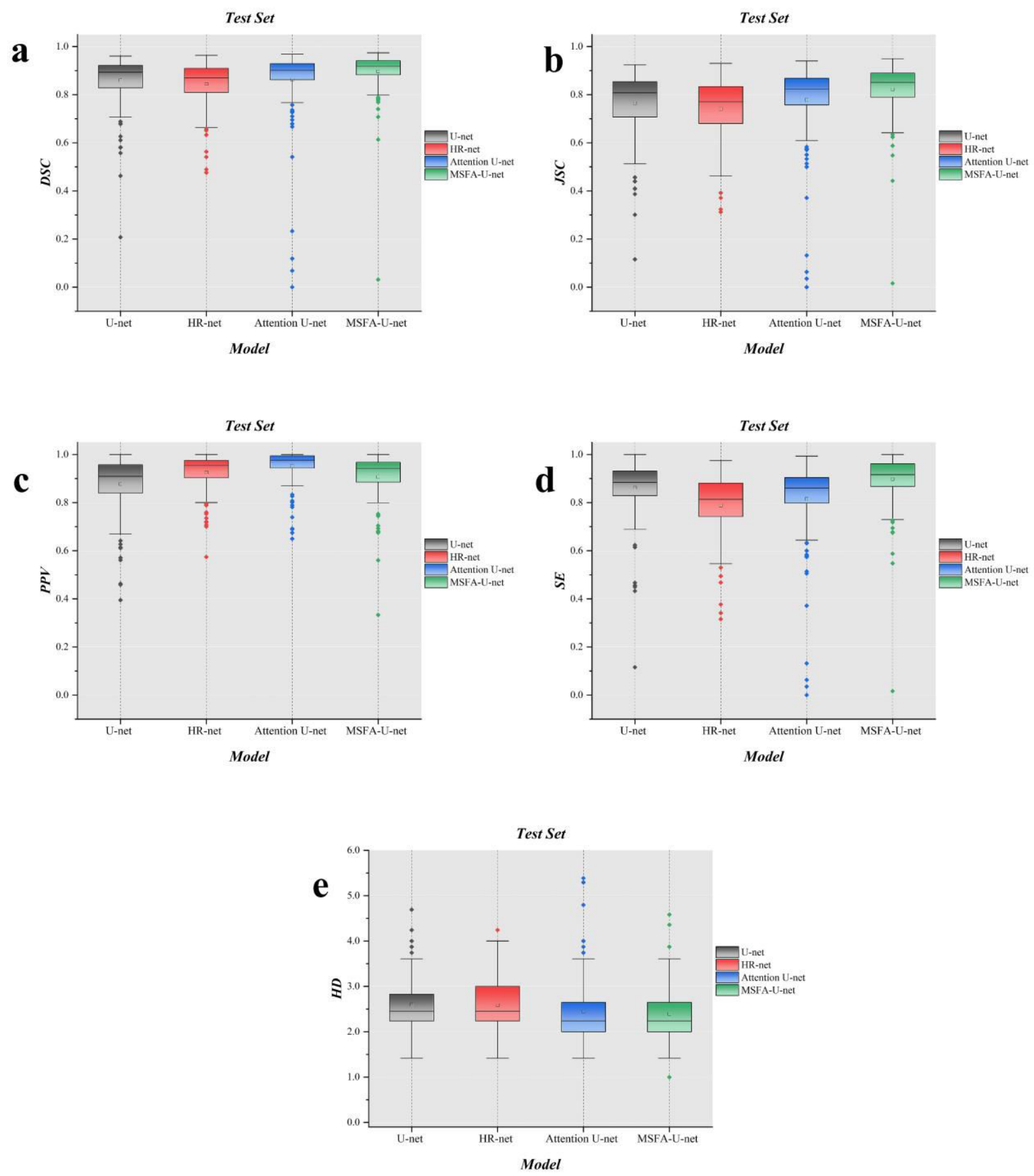

Fig. 6 Box plot diagrams in the test set (a. Box plot diagrams of DSC in the test set. b. Box plot 
Taken together, the Attention U-net (which introduces gated attention) and the HR-net did not reason may be that although the gated attention mechanism can effectively segment the target category and location, it may also lead to an increase in model false-positive predictions for small-volume segmentation with variability in shape. The HR-net performs multiple simple feature fusions; although it can effectively fuse features and obtain rich high-resolution features, it is also more likely to cause overfitting in the event of relatively scarce training data. Thus, the phenomenon of integration leads to a decline in its generalization ability. Clearly, the increase in model parameters and the increase in resource consumption may not necessarily improve the corresponding results.

\section{Discussion}

breast cancer. For the design and implementation of radiotherapy plans, accurate regulation of the radiation dose within the target area and limiting the dose to the surrounding OARs are important parts of the evaluation of a treatment plan. Precise delineation of OARs is important to effectively limit the dose outside the target area and avoid side effects of radiotherapy (27). The thyroid gland is an OAR during treatment of head and neck tumors and breast cancer, so it needs to be protected during radiotherapy. 
gland segmentation on CT localization images for radiotherapy. The innovation of this algorithm lies in the addition of multiple parallel channels on the basis of the traditional U-net model. It fully integrates the feature information between different resolutions, thereby avoiding the single information in the Unet downsampling process. In addition, our study also introduced the cSE attention mechanism, which inclined the model to the ROI during the training process. The experimental results showed that, compared with other similar representative segmentation algorithms, the proposed model improved both qualitative and quantitative results to a certain extent, and had better robustness and generalization. The image segmentation graphs revealed that MSFA-U-net effectively reduced oversegmentation and undersegmentation, and it achieved smoother edges. According to Zijdenbo et al. (28), DSC $>0.7000$ indicates that the segmentation meets the basic standard. While all of the models in this study reached this threshold, the DSC value of the MSFA-U-net reached 0.8967 , indicating that this model can effectively segment the thyroid gland on CT localization images for radiotherapy. From the box plot diagrams, it is evident that the MSFA-U-net yields good quantitative results, where the upper and lower quartile gaps and outliers of most evaluation indicators were reduced, indicating that the model achieves a consistent segmentation on the different layers of the thyroid gland and can effectively segment the thyroid gland on CT localization images for radiotherapy. However, there are some limitations in the algorithm proposed in this study, which needs to be further improved. Some of

281 the evaluation indicators of MSFA-U-net have not yet reached the optimal results. Moreover, the 282 introduction of a large number of feature fusions between different scales resulted in an increase in the amount of model parameters. In addition, although the Dice loss function can effectively solve the problem of class imbalance, its gradient characteristics may cause the model to oscillate during the training process. In future research, we will explore how to reduce the parameter count of the model 
while using different loss functions to further optimize the model.

\section{Conclusions}

In summary, the MSFA-U-net model makes it possible for radiologists to automatically delineate the thyroid gland on CT localization images for radiotherapy, and the results show that the model can be well applied to clinical work. Compared with the three commonly used models in the medical field, the MSFA-U-net model can offer more help to radiologists by delineating the thyroid gland more accurately and helping save time in delineation.

\section{List of abbreviations}

CT: computed tomography; MSFA-U-net: Multi-scale Fusion Attention U-net; DSC: Dice similarity coefficient; JSC: Jaccard similarity coefficient; PPV: positive predictive value; SE: sensitivity; HD: Hausdorff distance; HR-net: High-Resolution net; OARs: organs at risk; MRI: magnetic resonance imaging; 3D-CRT: three-dimensional conformal radiotherapy; 3D: three-dimensional; ROI: region of interest; cSE: spatial squeeze and channel excitation block; BN: batch normalization; GAP: global average pooling.

\section{Reference} 2018: GLOBOCAN estimates of incidence and mortality worldwide prostate cancers and their relationship with the human development index. Advances in Human Biology. 2019;9. 
changes in nasopharyngeal carcinoma patients in 48 months after radiotherapy. PLOS ONE. 2018;13:e0200310.

3. Buchholz T. Radiation after breast conservative surgery or after mastectomy reduces local recurrence and improves overall survival in breast cancerEarly Breast Cancer Trailists' Collaborative Group. Effects of radiotherapy and of differences in the extent of surgery for early breast cancer on local recurrence and 15-year survival: an overview of the randomized trials. Lancet 2005;366:20872106. The Women's Oncology Review. 2011;6.

4. Forastiere A, Zhang Q, Weber R, Maor M, Goepfert H, Pajak T, et al. Long-Term Results of RTOG 91-11: A Comparison of Three Nonsurgical Treatment Strategies to Preserve the Larynx in Patients With Locally Advanced Larynx Cancer. Journal of clinical oncology : official journal of the American Society of Clinical Oncology. 2012;31.

5. Ronneberger O, Fischer P, Brox T. U-Net: Convolutional Networks for Biomedical Image Segmentation2015. 234-41 p.

6. Hesamian MH, Jia W, He X, Kennedy P. Deep Learning Techniques for Medical Image Segmentation: Achievements and Challenges. Journal of Digital Imaging. 2019;32.

7. Ye Y, Cai Z, Huang B, He Y, Zeng P, Zou G, et al. Fully-Automated Segmentation of Nasopharyngeal Carcinoma on Dual-Sequence MRI Using Convolutional Neural Networks. Frontiers in Oncology. 2020;10:166.

8. Zhu W, Huang Y, Zeng L, Chen X, Liu Y, Qian Z, et al. AnatomyNet: Deep Learning for Fast and Fully Automated Whole-volume Segmentation of Head and Neck Anatomy. Medical Physics. 2018;46. segmentation. Journal of Medical Imaging. 2019;6. 
10. Zhang Z, Wu C, Coleman S, Kerr D. DENSE-INception U-net for medical image segmentation. Computer Methods and Programs in Biomedicine. 2020;192:105395.

11. Zhao N, Yang R, Jiang Y, Tian S, Guo F, Wang J. A hybrid IMRT/VMAT technique for the treatment of nasopharyngeal cancer. BioMed research international. 2015;2015:940102.

12. Franco A, Jolly LA, Russell S, Goldstein L, DeHart J. Abstract P5-06-01: The role of thyroid hormones in breast tumorigenesis: A translational study utilizing mouse models, cell culture and patient data. Cancer Research. 2016;76:P5-06.

13. Luo R, Wu V, He B, Gao X, Xu Z, Wang D, et al. Development of a normal tissue complication probability (NTCP) model for radiation-induced hypothyroidism in nasopharyngeal carcinoma patients. Bmc Cancer. 2018;18(1). Internal Covariate Shift. 2015. Medical Image Segmentation. 2016. 
20. Eelbode T, Bertels J, Berman M, Vandermeulen D, Maes F, Bisschops R, et al. Optimization for

Medical Image Segmentation: Theory and Practice When Evaluating With Dice Score or Jaccard Index.

21. Talluri S. Positive predictive value. BMJ. 2009;339:b3835-b.

22. Skaik Y. Understanding and using sensitivity, specificity and predictive values. Indian journal of ophthalmology. 2008;56:341; author reply

23. Kreveld M, Miltzow T, Ophelders T, Sonke W, Vermeulen J. Between shapes, using the Hausdorff distance. Computational Geometry. 2022;100:101817.

24. ke S, Xiao B, Liu D, Wang J. Deep High-Resolution Representation Learning for Human Pose Estimation2019.

25. Oktay O, Schlemper J, Folgoc L, Lee M, Heinrich M, Misawa K, et al. Attention U-Net: Learning Where to Look for the Pancreas. 2018.

Copyright $\neg$ C 2021, StatPearls Publishing LLC.; 2021.

27. Peng Y-L, Chen 1, Shen G-Z, Li Y-N, Yao J-J, Xiao W-W, et al. Interobserver variations in the modulated radiation therapy for nasopharyngeal carcinoma. Oral oncology. 2018;82:1-7. MR images. IEEE transactions on medical imaging. 1994;13:716-24.

\section{Declarations}


375 Institutional Review Board approval was not required because in order to protect the privacy of the patients, all the CT images in this study, when they were copied from the image library, were modified to delete the private information of the patients such as name, age, diagnosis, etc. This is a scientific study and isn't aimed at diagnosis and treatment, causing no harm to patients.

\section{Consent for publication}

$380 \quad$ Not applicable

\section{Availability of data and materials}

382 The datasets generated and/or analysed during the current study are not publicly available due patient confidentiality, but are available from the corresponding author on reasonable request.

\section{Competing interests}

The authors of this manuscript declare no relationships with any companies, whose products or services may be related to the subject matter of the article

\section{Funding}

This study has received funding by Climbing Foundation of National Cancer Center

(NCC201925B03).

\section{Authors' contributions}

All authors were involved in the conception of this study, design, and implementation. XBW was a major contributor in designing the models, drawing the figures and writing

393 the manuscript and was also involved in delineating some label images. YY performed the

394 checking and proofing of the manuscript and the data apart from guidance to the writing of the manuscript. BZ, MFY, JZL, MZS, LSM, and CXS were major contributors in data collection, delineation of most of the label images and output of data. All authors read and approved the final manuscript. 
398

399

400 manuscript.

402

\section{Acknowledgements}

We thank LetPub (www.letpub.com) for its linguistic assistance during the preparation of this

401 